Volume 3

$11-11-2016$

\title{
Determining the Incidence and Factors of Cardiotoxicity in Breast Cancer Patients Treated With Anthracycline and/or Trastuzumab- Containing Regimen at Aurora Health Care
}

Stephanie Ghojallu

Matthew Rappelt

Han-Yang Chen

Ruth Perez

Lydia Garlie

Geoffrey Riddell

Yingying $\mathrm{Gu}$

Jun Zhang

Rubina Qamar

Bijoy K. Khandheria

See next page for additional authors Follow this and additional works at: https://aah.org/jpcrr

Part of the Cardiology Commons, Clinical Epidemiology Commons, Neoplasms Commons, Oncology Commons, and the Therapeutics Commons

\section{Recommended Citation}

Ghojallu S, Rappelt M, Chen HY, Perez R, Garlie L, Riddell G, Gu Y, Zhang J, Qamar R, Khandheria BK, Jahangir $A$, Shi Y. Determining the incidence and factors of cardiotoxicity in breast cancer patients treated with anthracycline and/or trastuzumab-containing regimen at Aurora Health Care. J Patient Cent Res Rev. 2016;3:244.

Published quarterly by Midwest-based health system Advocate Aurora Health and indexed in PubMed Central, the Journal of Patient-Centered Research and Reviews (JPCRR) is an open access, peer-reviewed medical journal focused on disseminating scholarly works devoted to improving patient-centered care practices, health outcomes, and the patient experience. 
Determining the Incidence and Factors of Cardiotoxicity in Breast Cancer

Patients Treated With Anthracycline and/or Trastuzumab-Containing Regimen at Aurora Health Care

\section{Authors}

Stephanie Ghojallu, Matthew Rappelt, Han-Yang Chen, Ruth Perez, Lydia Garlie, Geoffrey Riddell, Yingying Gu, Jun Zhang, Rubina Qamar, Bijoy K. Khandheria, Arshad Jahangir, and Yang Shi 
Methods: We included 9 patients with typical atrial flutter for whom mapping and ablation of the CTI was done using Carto mapping as the sole guiding modality. Zero fluoroscopy was achieved in all of them. Another group of 9 matched patients whose ablations were done using the traditional method (i.e. fluoroscopy and Carto guidance) were included as a control.

Results: Both groups had similar baseline characteristics. The total fluoroscopy time and radiation dose in the control group were $12.8 \pm 4.8$ minutes, $230.2 \pm 131.9 \mathrm{mGy}$, respectively. The zero-fluoroscopy group were done while wearing no lead aprons. The average total procedure time was $114.8 \pm 16.9$ minutes in the zero-fluoroscopy group, significantly less than that of the control group $(138.6 \pm 24.3$ minutes; $\mathrm{P}=0.0286)$. The total radiofrequency time was similar in both groups $(15.2 \pm$ 7.4 minutes in zero-fluoroscopy group vs $16 \pm 2.9$ minutes in control group; $\mathrm{P}=0.9294)$. Bidirectional block was achieved in all patients of both groups. No complications were encountered in either group.

Conclusion: To our knowledge, this is the first study of a zerofluoroscopic approach using the Carto mapping system for ablation of the cavotricuspid isthmus in the United States. The approach is feasible and effective in achieving bidirectional block with less average total procedure time.

\section{Determining the Incidence and Factors of Cardiotoxicity in Breast Cancer Patients Treated With Anthracycline and/or Trastuzumab-Containing Regimen at Aurora Health Care}

Stephanie Ghojallu, Matthew Rappelt, Han-Yang Chen, Ruth Perez, Lydia Garlie, Geoffrey Riddell, Yingying Gu, Jun Zhang, Rubina Qamar, Bijoy K. Khandheria, Arshad Jahangir, Yang Shi

Aurora Nursing Administration; Aurora Research Institute; University of Wisconsin-Milwaukee; Hematology/ Oncology, Aurora Health Care; Aurora Cardiovascular Services and Sheikh Khalifa bin Hamad Al Thani Center for Integrative Research on Cardiovascular Aging, Aurora Health Care

Background: Anthracycline and trastuzumab are common breast cancer treatments. While improving survival, they elevate risk of congestive heart failure. The incidence of cardiotoxicity (CTx) with these therapies varies in the literature from $10 \%$ to $59 \%$, higher than those reported in clinical trials $(4 \%-$ $10 \%)$ that excluded patients with preexisting cardiovascular comorbidities. Studies have failed to establish consensus on the risk factors for CTx associated with these therapies.

Purpose: We aim to determine the incidence and risk factors of CTx in breast cancer patients treated with anthracycline and/or trastuzumab at Aurora Health Care.

Methods: A retrospective review of patients with breast cancer who received anthracycline and/or trastuzumab from 2002 to 2011 yielded a total of 2,383 patients. Patients with a left ventricular ejection fraction (LVEF) recorded prior to treatment and at least one follow-up LVEF were included in analysis ( $n=319,13.4 \%$ of total cohort). Database queries and electronic medical records review (assisted by an inhouse natural language processing tool) retrieved data on demographics, comorbidities, congestive heart failure symptoms, oncological treatments and LVEF. The study outcome was CTx, defined as a $\geq 10 \%$ decrease in LVEF to a level of $<55 \%$. Chi-squared and Fisher's exact tests were used for categorical variables to test differences in patient characteristics by CTx status (yes/no). Multivariate logistic regression analyses examined the association between risk factors and CTx.

Results: Average age of the patients was $54.9 \pm 12.1$ years; the cohort was comprised of $50.5 \%$ with obesity, $44.2 \%$ with smoking history and $47.3 \%$ with hypertension. A total of 79 patients developed CTx, an incidence of $24.8 \%$. Multivariable analysis identified divorced/widowed marital status (odds ratio [OR]: 2.70, 95\% confidence interval [CI]: 1.26-5.77), history of structural/electrophysiological (EP) cardiac disease (OR: 2.66, 95\% CI: 1.24-5.70) and combined anthracycline-trastuzumab therapy (OR: 2.92, 95\% CI: 1.48-5.77) as significant risk factors for CTx.

Conclusion: The incidence of CTx was greater in a community setting for which cardiac history and comorbidities are more diverse than in clinical trials. Consistent with prior literature, our study identified combined treatment with anthracycline and trastuzumab as a risk factor for CTx. Our study also suggests divorced/widowed marital status and prior structural/EP cardiac disease as additional risk factors for CTx. Further prospective studies are warranted for verification. We advocate for pre- and posttreatment cardiac monitoring of patients receiving these two therapies.

\section{Are There Advantages to Hiring In-House Training Program Graduates?}

Jessica J.F. Kram, Dennis J. Baumgardner

Department of Family Medicine, Aurora UW Medical Group; Center for Urban Population Health

Background: Several studies have compared international graduates on measures of performance, quality and satisfaction. No studies have compared internally versus externally hired graduates in relation to these measures.

Purpose: To identify if there is a difference in hiring patterns and care management (CM)/patient satisfaction (PS) scores between internal and external graduate hires.

Methods: We conducted a quality improvement study on graduates hired by Aurora Health Care from Jan. 1, 2006, to Dec. 14, 2015. CM scores were determined based on hire date. PS scores were calculated based on the calendar year, regardless of exact hire date. PS scales for scoring changed in mid-2010. Hired graduates with no CM and PS scores, as well as those with less than one year of employment, were excluded. Means were compared using two-sample t-tests and regression analysis. Categorical variables were analyzed using chi-squared and Fisher's exact test, as appropriate.

Results: Study population $(\mathrm{N}=108)$ characteristics included: mean age 38.0 years, $62.0 \%$ female gender and $56.5 \%$ 